\title{
A study of cognitive function in treatment-refractory obsessive-compulsive disorder treated with capsulotomy
}

\author{
*Feilong Gong, MD,, Peng Li, MD, PhD, ${ }^{1}$ Bin Li, MD, PhD, ${ }^{2}$ Shizhen Zhang, MD, ${ }^{1}$ Xinjie Zhang, MD, ${ }^{1}$ \\ Sen Yang, MD, ${ }^{1}$ Hongbin Liu, MD, ${ }^{1}$ and Wei Wang, MD, PhD'1 \\ Departments of ${ }^{1}$ Neurosurgery and ${ }^{2}$ Psychiatry, West China Hospital, Sichuan University, Chengdu, Sichuan Province, People's \\ Republic of China
}

\begin{abstract}
OBJECTIVE Anterior capsulotomy (AC) is sometimes used as a last resort for treatment-refractory obsessive-compulsive disorder $(\mathrm{OCD})$. Previous studies assessing neuropsychological outcomes in patients with OCD have identified several forms of cognitive dysfunction that are associated with the disease, but few have focused on changes in cognitive function in OCD patients who have undergone surgery. In the present study, the authors investigated the effects of AC on the cognitive function of patients with treatment-refractory OCD.
\end{abstract}

METHODS The authors selected 14 patients with treatment-refractory OCD who had undergone bilateral AC between 2007 and 2013, 14 nonsurgically treated OCD patients, and 14 healthy control subjects for this study. The 3 groups were matched for sex, age, and education. Several neuropsychological tests, including Similarities and Block Design, which are subsets of the Wechsler Abbreviated Scale of Intelligence; Immediate and Delayed Logical Memory and Immediate and Delayed Visual Reproduction, which are subsets of the Wechsler Memory Scale-Revised; and Corrects, Categories, Perseverative Errors, Nonperseverative Errors, and Errors, subtests of the Wisconsin Card Sorting Test, were conducted in all 42 subjects at baseline and after AC, after nonsurgical treatment, or at 6-month intervals, as appropriate. The Yale-Brown Obsessive-Compulsive Scale (Y-BOCS) was used to measure OCD symptoms in all 28 OCD patients.

RESULTS The Y-BOCS scores decreased significantly in both OCD groups during the 12-month follow-up period. Surgical patients showed higher levels of improvement in verbal memory, visual memory, visuospatial skills, and executive function than the nonsurgically treated OCD patients.

CONCLUSIONS The findings of this study suggest that AC not only reduces OCD symptoms but also attenuates moderate cognitive deficits.

https://thejns.org/doi/abs/10.3171/2016.9.JNS152494

KEY WORDS anterior capsulotomy; treatment-refractory obsessive-compulsive disorder; neuropsychology; functional neurosurgery

$\mathrm{U}$ P to $10 \%$ of patients with obsessive-compulsive disorder (OCD) fail to respond to first-line pharmacological and psychotherapeutic treatments and thus are considered to have treatment-refractory disease, ${ }^{17}$ which is often defined as a minimal or absent response to an adequate number of trials of selective serotonin reuptake inhibitors (SSRIs; minimum 3 types of SSRI at maximum dosage for at least 12 weeks), standard augmentation strategies (2 atypical antipsychotics), and behavior thera- py (minimum 20 hours). ${ }^{12}$ Anterior capsulotomy (AC) is a neurosurgical option for treatment-refractory OCD, after which many patients experience remarkable clinical improvement. ${ }^{16,21,28,34}$ During AC, lesions are introduced in the anterior limb of the internal capsule. These lesions extend across the anterior thalamic projection fibers and interfere with the fibers connecting the mediodorsal thalamus and the prefrontal cortex. The mechanism underlying this neurosurgical method may be related to the cortico-

ABBREVIATIONS AC = anterior capsulotomy; CBT = cognitive behavioral therapy; DSM = Diagnostic and Statistical Manual of Mental Disorders; GVC = gamma ventral capsulotomy; $\mathrm{OCD}$ = obsessive-compulsive disorder; RCT = randomized controlled trial; $S 1, \ldots$ S14 = surgical patient $1, \ldots$ surgical patient $14 ;$ SSRI = selective serotonin reuptake inhibitor; VC/VS = ventral capsular/ventral striatal; WASI = Wechsler Abbreviated Scale of Intelligence; $W C S T=$ Wisconsin Card Sorting Test; WMS-R = Wechsler Memory Scale-Revised; Y-BOCS = Yale-Brown Obsessive Compulsive Scale.

SUBMITTED October 28, 2015. ACCEPTED September 28, 2016.

INCLUDE WHEN CITING Published online March 24, 2017; DOI: 10.3171/2016.9.JNS152494.

* Drs. Gong and P. Li contributed equally to this work. 
striato-thalamo-cortical loop, which is hypothesized to be dysfunctional in OCD. However, several adverse effects are associated with $\mathrm{AC}$, most commonly cognitive functional impairments such as memory problems and executive dysfunction..$^{25,26,34}$ However, it is worth noting that very few studies have systematically assessed changes in cognitive performance following AC. 3,7,42

At the Karolinska Hospital in Stockholm between 1978 and 1990, Nyman et al. ${ }^{25}$ observed 21 patients with extreme OCD treated with thermocapsulotomy. Another 8 OCD patients seen more recently served as a control group. At follow-up, all patients were examined with a comprehensive neuropsychological test battery including the Wechsler Adult Intelligence Scale-Revised, Controlled Oral Word Association Test, Swedish Claeson-Dahl Test, Rey Complex Figure Test, and the Wisconsin Card Sorting Test (WCST). In a subgroup of surgically treated patients who did not complete all subtests on the WCST, there was a significant positive correlation between time elapsed after capsulotomy and number of subtests completed on the WCST. Researchers found that patients with extreme OCD as a group perform in the lower to mildly impaired range on neuropsychological tests. In some patients, executive function was affected but restored over time.

Rück et al. ${ }^{34}$ examined 25 OCD patients who had undergone AC between 1988 and 2000 at the Karolinska Institute in Stockholm, Sweden. Results of pre- and postoperative neuropsychological tests, including the Wechsler Adult Intelligence Scale-Revised, Controlled Oral Word Association Test, WCST, and simplified Six Elements Task, were recorded for 7 patients. The pre- and postoperative data from the WCST and Digit Span Test suggested more compromised executive function at the long-term followup. The study results suggested that clinical and cognitive improvement stagnate after 1 year postoperatively, and the authors concluded that there were no significant differences between the 1-year and the long-term follow-up ratings, implying that improvement was generally stable.

Taub et al. ${ }^{42}$ observed 5 patients with severe OCD who had undergone ventral capsular/ventral striatal (VC/VS) gamma capsulotomy between 2003 and 2004 at the Santa Paula Hospital in Brazil. The neuropsychological battery consisted of attention (Trail Making Test A and B), executive function (Block Design subtest and Trail Making Test B), and memory (Rey Auditory Verbal Learning Test, Logical Memory subtests of the Wechsler Memory ScaleRevised [WMS-R]) measures. To evaluate intellectual function, the authors obtained scores from the Wechsler Abbreviated Scale of Intelligence (WASI), including the Vocabulary, Similarities, Matrix Reasoning, and Block Design subtests. At the 12-month follow-up, improvements in attention, vocabulary, learning, abstract reasoning, and memory had occurred.

At Gyula Nyíró Hospital in Budapest, Hungary, Csigó et al. ${ }^{7}$ examined 10 patients with treatment-refractory OCD who were divided into 2 groups (surgical and nonsurgical) of 5 participants each. Ten neuropsychological tests were used to measure cognitive function. At the 2-year follow-up, the investigators found a significant increase in most of the neuropsychological test scores in both surgical (thermocapsulotomy) and nonsurgical groups. They observed intrusion errors only on the Category Fluency Test. The nonsurgical group showed better performance than the surgical group on only 3 tests: Trail Making Test B, Repeatable Battery for the Assessment of Neuropsychological Status (RBANS) Attention Index, and RBANS Language Index. The clinical and neuropsychological improvements were more impressive in the surgical group.

At the Santa Paula Hospital in Brazil, Batistuzzo et al. ${ }^{3}$ conducted, in the first phase of the trial, a double-blind randomized controlled trial (RCT) of gamma ventral capsulotomy (GVC) in 16 patients with refractory OCD. The secondary analysis phase included 17 patients: 8 from the active treatment group, 4 from the sham group who underwent surgery after the blind phase was over, and 5 patients from a previous open pilot study. A comprehensive neuropsychological evaluation of intellectual functioning, attention, verbal and visuospatial learning and memory, visuospatial perception, inhibitory control, cognitive flexibility, and motor functioning was performed at baseline and at the 1-year follow-up. In the RCT, visuospatial memory performance significantly improved in the active treatment group after GVC and remained stable in the sham group. There was no decline in cognitive or motor functioning after 1 year in the surgically treated patients. The authors concluded that improvement in certain neuropsychological domains, particularly visuospatial memory performance, occurred 1 year after GVC in patients with treatment-refractory OCD.

Currently, there are no reliable conclusions regarding neuropsychological changes after AC. Most recent studies have shown that patients experience some degree of improvement in cognitive functioning domains such as intellectual functioning (IQ), verbal memory, visuospatial memory, and executive functioning. ${ }^{3,7,42}$ However, given the lack of healthy control subjects in these studies, the degree and type of cognitive impairments experienced by patients before surgery, as well as the extent to which these impairments are improved by surgery, are not entirely clear. Moreover, few studies have analyzed the variations in cognition at different follow-up time points. Therefore, in the present study, we included a nonsurgical OCD group, as well as a healthy control group, each comprising 14 subjects. Our aim was to investigate changes in cognitive function over time following $\mathrm{AC}$ in patients with treatment-refractory OCD. We hypothesized that OCD patients would exhibit poorer cognitive performance than healthy controls at baseline but would improve over the 12 months after AC. Furthermore, we anticipated that OCD patients would exhibit normal performance levels on several cognitive tests.

\section{Methods}

\section{Inclusion and Exclusion Criteria, Ethical Issues, and Demographics}

Diagnoses were made by 2 psychiatrists using the criteria of the Diagnostic and Statistical Manual of Mental Disorders (DSM-IV or DSM-5). The treatment-refractory nature of the illness was carefully defined by the following criteria: meeting the DSM-IV or DSM-5 criteria for a diagnosis of OCD; having undergone systematic treat- 
ment with at least 3 different trials of SSRIs and an adequate drug therapy duration of a minimum of 12 weeks; having participated in cognitive behavioral therapy (CBT) for more than 20 hours (or an inability to participate in CBT because of severe OCD symptoms); and having a less than 25\% reduction in Yale-Brown Obsessive Compulsive Scale (Y-BOCS) scores after both drug therapy and CBT.12 Inclusion criteria for the surgical OCD patients were as follows: an age between 18 and 60 years, a 5-year history of obsessive-compulsive symptoms causing substantial suffering and significantly reduced functioning, meeting the DSM-IV or DSM-5 criteria for the diagnosis of OCD, and disease identified as treatment refractory by the 2 aforementioned psychiatrists. Consent from both the patients and their families was required for surgical treatment. Patients with a history of head injury, posttraumatic amnesia, brain tumor, or other somatic disease that precluded surgery were excluded from the study, as were those who were unable to give informed consent, as determined via neuropsychological testing. ${ }^{12}$ This study was approved by the institutional review board of the West China Hospital of Sichuan University Clinical Trials and Biomedical Ethics Committee. All patients signed informed consent forms.

Randomized surgical OCD patient selection was not possible due to ethical concerns; therefore, we performed a retrospective nonrandomized study. We selected 14 consecutive patients from a group of 38 treatment-refractory OCD patients who had undergone AC via thermocoagulation between 2007 and 2013 at the West China Hospital. To minimize the effects of confounding variables, we selected 14 age-, sex-, and Y-BOCS score-matched nonsurgical OCD patients who had been evaluated at the same center between 2008 and 2013. All surgical and 3 nonsurgical OCD patients were, based on the abovementioned criteria, diagnosed with treatment-refractory disease by the 2 aforementioned psychiatrists. Moreover, we included 14 age- and sex-matched healthy volunteers who had undergone the same neuropsychological examinations by the same psychiatrists between 2012 and 2014 . The demographic characteristics of all 42 subjects and the clinical symptoms of the patients in the 2 OCD groups are shown in Table 1. There were no differences among the 3 groups with respect to age (chi-square $=0.451, \mathrm{p}=0.798$ ), education level (chi-square $=0.117, \mathrm{p}=0.943$ ), sex (male/ female: $5 / 9,5 / 9,6 / 8$ ), and handedness (all patients were right handed). The surgical and nonsurgical groups did not differ significantly regarding Y-BOCS scores $(\mathrm{z}=-0.554$, $p=0.580)$ or hours of CBT $(z=-0.184, p=0.854)$ at baseline but did differ in terms of OCD duration $(z=-3.441$, $\mathrm{p}=0.001)$ and SSRI treatment duration $(\mathrm{z}=-3.892, \mathrm{p}=$ 0.000 ). Eight patients were unemployed, and 6 patients in the surgery group had previously attempted suicide.

\section{Clinical Assessments and Neuropsychological Tests}

All 42 subjects underwent face-to-face assessments performed by the 2 previously mentioned psychiatrists at our medical center at baseline, as well as at regular intervals during a 12-month follow-up. No surgical patients received any drugs or CBT during the 12-month follow-up period, except for 3 patients who received limited and discontinuous drug treatment because of limited postsurgi- cal improvement. It was not possible to ensure that every patient in the nonsurgical group complied with the treatment regimens (in terms of medication dosages and CBT time) throughout the 12-month follow-up period; however, every nonsurgical patient received adequate dosages of SSRIs (one of which was clomipramine) and underwent CBT for more than 2 hours per week with the same therapist during the follow-up. We used the Y-BOCS ${ }^{9}$ to assess obsessive-compulsive symptoms in the OCD patients at the indicated time points (baseline and 1, 3, 6, and 12 months after treatment). Patients were considered to be responsive to therapy if they had a Y-BOCS score reduction of $35 \%$ or more as compared with their baseline score. Given concerns regarding patient adherence to therapeutic regimens as well as their ability to complete complex comprehensive tests, we made the neuropsychological evaluation as simple as possible and used specific subtests. Each test session lasted approximately 50 minutes or less for each participant. The neuropsychological battery consisted of the following measures: Similarities and Block Design, which are subtests of the WASI; Immediate and Delayed Logical Memory and Immediate and Delayed Visual Reproduction, subtests of the WMS-R; and Corrects, Categories, Perseverative Errors, Nonperseverative Errors, and Errors, subtests of the WCST. The neuropsychological evaluations in the OCD patients were performed at the same time points as the Y-BOCS examinations (baseline and 1,3,6, and 12 months after treatment). The domains and functions for each subtest are shown in Table 2. To minimize placebo effects, we used different versions of each subtest at different time points, if possible (for example, Logical Memory Tests A and B and Visual Reproduction Tests A, B, and C). For cognitive assessment in the healthy control group, we adopted follow-up intervals of 6 months. In addition, CT was performed 1 day after surgery in the surgical group and MRI was performed 3 months after surgery to minimize the effects of early edema or bleeding on imaging results. Functional diffusion tensor imaging ( 1 day before surgery and 3 and 6 months after surgery) and PET/CT (1 day before surgery and 6 and 12 months after surgery) were performed in 14 surgical OCD patients to evaluate changes in cerebral white matter fiber and metabolism. Representative MRI results from 3 months after surgery are shown in Fig. 1.

\section{Surgical Technique of AC}

For optimal targeting, MR images without fixation of the head frame were obtained the day before surgery for preplanning. A 3-T Trio Unite MRI system (Siemens AG) was used with T1-weighted and T2-weighted spin-echo sequences in the axial and coronal planes. Slice thickness was $2 \mathrm{~mm}$ with an interval gap of $0.02 \mathrm{~mm}$. The preplanning procedure was completed using the SurgiPlan workstation (version 2.1, Elekta Instruments AB). On the day of surgery, a Leksell model G head frame (Elekta Instruments $\mathrm{AB}$ ) was positioned on the skull parallel to the anterior commissure-posterior commissure line with the patient under local anesthesia. Repeat MRI with the same parameters applied in the preplanning scanning was performed using a 1.5-T Sonata Unite MRI system (Siemens AG). With the aid of the SurgiPlan workstation, the pre- 
TABLE 1. Baseline demographic characteristics of the 3 groups and the clinical features of the OCD patients included in this study*

\begin{tabular}{|c|c|c|c|c|c|c|c|c|}
\hline $\begin{array}{c}\text { Patient No. \& } \\
\text { Demographics }\end{array}$ & $\begin{array}{l}\text { Age } \\
\text { (yrs) }\end{array}$ & $\begin{array}{c}\text { Duration } \\
\text { of OCD } \\
\text { (yrs) }\end{array}$ & $\begin{array}{c}\text { Yrs } \\
\text { of } \\
\text { Education }\end{array}$ & $\begin{array}{c}\text { SSRI } \\
\text { Treatment } \\
\text { Duration } \\
\text { (yrs) }\end{array}$ & $\begin{array}{l}\text { Hrs } \\
\text { of } \\
\text { CBT }\end{array}$ & $\begin{array}{l}\text { Y-BOCS } \\
\text { Score }\end{array}$ & $\begin{array}{l}\text { Concomitant } \\
\text { Disorder }\end{array}$ & Job \\
\hline \multicolumn{9}{|l|}{ Surgical group $(n=14)$} \\
\hline S1†‡ & 30 & 7 & 16 & 3 & 35 & 22 & None & Unemployed \\
\hline$S 2^{*} \ddagger$ & 42 & 24 & 11 & 1 & NA & 28 & GAD & Official \\
\hline S3†‡ & 49 & 32 & 16 & 22 & 40 & 32 & MDD, GAD & Businessman \\
\hline$S 4^{*} \S$ & 23 & 10 & 9 & 5 & 30 & 26 & GAD & Professional \\
\hline$S 5^{*} \S$ & 23 & 6 & 11 & 3 & 60 & 32 & GAD & Waiter \\
\hline$S 6 * \ddagger$ & 29 & 11 & 15 & 11 & 55 & 36 & MDD, GAD & Cashier \\
\hline S7†§ণ & 18 & 5 & 9 & 4 & 80 & 32 & $\mathrm{GAD}, \mathrm{SP}$ & Unemployed \\
\hline S8†§ & 31 & 10 & 15 & 6 & 40 & 28 & GAD & Unemployed \\
\hline s9†‡ & 29 & 17 & 9 & 8 & 45 & 25 & GAD & Driver \\
\hline $\mathrm{S} 10^{*} \S$ & 21 & 10 & 14 & 6 & 33 & 30 & GAD, TWAD & College student \\
\hline $\mathrm{S} 11^{*} \S$ & 39 & 5 & 16 & 15 & 35 & 18 & MDD & Unemployed \\
\hline$S 12^{*} \mp \mathbb{T}$ & 57 & 30 & 6 & 28 & NA & 35 & MDD, GAD & Businessman \\
\hline $\mathrm{S} 13^{*} \S$ & 23 & 5 & 14 & 4 & 48 & 28 & GAD & Unemployed \\
\hline S14*§T & 20 & 6 & 12 & 5 & 60 & 37 & GAD, OS, MDD & College dropout \\
\hline Median & 29 & 10.0 & 13.0 & 5.0 & 40 & 29.0 & & \\
\hline Mean & 31.0 & 12.6 & 12.4 & 8.7 & 40.1 & 29.0 & & \\
\hline SD & 3.1 & 10.0 & 0.9 & 2.3 & 21.7 & 1.0 & & \\
\hline$p$ value & 0.798 & 0.001 & 0.943 & 0.000 & 0.854 & 0.580 & & \\
\hline \multicolumn{9}{|c|}{ Nonsurgical group $(n=14)$} \\
\hline NS1†§ & 32 & 2 & 11 & 1 & 40 & 32 & GAD & Official \\
\hline NS2* $\ddagger$ & 35 & 1 & 16 & 0.5 & 36 & 40 & MDD, GAD & Teacher \\
\hline NS3†§ & 20 & 1 & 12 & 0.5 & NA & 39 & GAD & College student \\
\hline NS4†§』 & 19 & 2 & 9 & 1 & 36 & 38 & MDD, GAD & College dropout \\
\hline NS5*§ & 27 & 1 & 12 & 0.8 & 58 & 33 & GAD & College student \\
\hline NS6+§ & 24 & 6 & 14 & 3 & 32 & 32 & GAD & Soldier \\
\hline NS7†§ & 19 & 3 & 9 & 2 & 45 & 39 & MDD, GAD & College student \\
\hline$N S 8^{*} \S$ & 19 & 3 & 11 & 1.5 & 72 & 31 & GAD & College student \\
\hline NS9* $\ddagger$ & 34 & 1 & 13 & NA & 50 & 25 & GAD & Staff member \\
\hline NS10* $\neq \mathbb{1}$ & 36 & 3 & 9 & 2 & NA & 26 & MDD, TWAD & Unemployed \\
\hline $\mathrm{NS} 11^{*} \neq \mathbb{I}$ & 28 & 2 & 7 & 1 & 30 & 27 & MDD & Unemployed \\
\hline NS12* $\ddagger$ & 37 & 1 & 16 & 0.3 & 50 & 21 & None & Mechanic \\
\hline NS13* $\ddagger$ & 27 & 13 & 16 & 10 & 49 & 26 & GAD & Official \\
\hline NS14†§ & 34 & 11 & 16 & 8 & 35 & 22 & MDD & Official \\
\hline Median & 27.5 & 2.0 & 12.0 & 1.0 & 38 & 29.0 & & \\
\hline Mean & 27.9 & 3.6 & 12.2 & 2.4 & 38.1 & 29.0 & & \\
\hline SD & 1.8 & 1.0 & 0.8 & 0.8 & 19.7 & 7.0 & & \\
\hline \multicolumn{9}{|c|}{ Healthy control group $(n=14)$} \\
\hline $\mathrm{HC}^{*} \ddagger$ & 52 & & 9 & & & & & Cleaner \\
\hline $\mathrm{HC} 2 \dagger \ddagger$ & 50 & & 12 & & & & & Mechanic \\
\hline $\mathrm{HC}^{*} \ddagger$ & 40 & & 9 & & & & & Dormitory admin \\
\hline $\mathrm{HC}^{*} \ddagger$ & 35 & & 9 & & & & & Housewife \\
\hline HC5†‡ & 30 & & 16 & & & & & Official \\
\hline $\mathrm{HC6}^{*} \ddagger$ & 26 & & 15 & & & & & Staff member \\
\hline $\mathrm{HC} 7+\pi$ & 25 & & 17 & & & & & College student \\
\hline
\end{tabular}


» CONTINUED FROM PAGE 586

TABLE 1. Baseline demographic characteristics of the 3 groups and the clinical features of the OCD patients included in this study*

\begin{tabular}{|c|c|c|c|c|c|c|c|c|}
\hline $\begin{array}{l}\text { Patient No. \& } \\
\text { Demographics }\end{array}$ & $\begin{array}{l}\text { Age } \\
\text { (yrs) }\end{array}$ & $\begin{array}{c}\text { Duration } \\
\text { of OCD } \\
\text { (yrs) }\end{array}$ & $\begin{array}{c}\text { Yrs } \\
\text { of } \\
\text { Education }\end{array}$ & $\begin{array}{c}\text { SSRI } \\
\text { Treatment } \\
\text { Duration } \\
\text { (yrs) }\end{array}$ & $\begin{array}{l}\mathrm{Hrs} \\
\text { of } \\
\text { CBT }\end{array}$ & $\begin{array}{l}\text { Y-BOCS } \\
\text { Score }\end{array}$ & $\begin{array}{l}\text { Concomitant } \\
\text { Disorder }\end{array}$ & Job \\
\hline \multicolumn{9}{|l|}{$\begin{array}{l}\text { Healthy control group } \\
\text { (continued) }\end{array}$} \\
\hline $\mathrm{HC}^{*} \ddagger$ & 30 & & 14 & & & & & Teacher \\
\hline $\mathrm{HCg}^{*} \boldsymbol{\pi}$ & 17 & & 10 & & & & & Student \\
\hline HC10*ף & 21 & & 13 & & & & & Waiter \\
\hline HC11†ף & 18 & & 11 & & & & & Soldier \\
\hline $\mathrm{HC12†T}$ & 19 & & 9 & & & & & Unemployed \\
\hline $\mathrm{HC13}^{*} \mathbb{I}$ & 21 & & 12 & & & & & College student \\
\hline $\mathrm{HC} 14 \dagger \S$ & 20 & & 12 & & & & & College student \\
\hline Median & 25.5 & & 12.0 & & & & & \\
\hline Mean & 28.9 & & 12.0 & & & & & \\
\hline SD & 3.1 & & 0.7 & & & & & \\
\hline \multicolumn{9}{|c|}{$\begin{array}{l}\text { Admin = administrator; } \mathrm{GAD}=\text { generalized anxiety disorder; } \mathrm{HC}=\text { healthy control subject; } \mathrm{MDD}=\text { major depressive disorder; } \mathrm{NA}=\text { not appli- } \\
\text { cable; } \mathrm{NS}=\text { nonsurgical patient; } \mathrm{OS}=\text { obsessive slowness; } \mathrm{S}=\text { surgical patient; } \mathrm{SP}=\text { schizophrenia; } \mathrm{TWA}=\text { two-way affective disorder. } \\
\text { * Female. } \\
\text { † Male. } \\
\text { † Married. } \\
\text { § Single. } \\
\text { † Unable to care for self. }\end{array}$} \\
\hline
\end{tabular}

scanned images were coregistered with the images using location markers. The coordinates of the target and the angles of electrode penetration were calculated. Lesion targets were located $14 \mathrm{~mm}$ anterior and $18 \mathrm{~mm}$ lateral to the anterior commissure and $5 \mathrm{~mm}$ below the anterior commissure-posterior commissure plane. Under the guidance of the Leksell multifunctional stereotactic operation system (Elekta Instruments AB), we inserted the electrode into the lesion target according to the calculated coordinates. Test stimulation generated by the Elekta neurostimulator at both high $(130 \mathrm{~Hz})$ and low $(5 \mathrm{~Hz})$ frequency was performed to verify the target of the electrode. Next, lesions were produced using the Elekta neurostimulator at $75^{\circ} \mathrm{C}$ for 60 seconds each. The length of the lesions on both sides was $12-14 \mathrm{~mm}$.

\section{Data Analysis}

All data were analyzed using SPSS software (SPSS 19.0 for Windows, IBM Corp.). Considering the small sample size and the particularly nonnormal distribution of the data, we used nonparametric tests. Demographic characteristics and baseline clinical features were compared between the OCD and control groups using the Kruskal-Wallis test and the Mann-Whitney U-test. Repeated measures ANOVAs were performed using "time" (baseline and 1, 3,6, and 12 months after surgery) as the within-subjects factor in both the surgical and nonsurgical groups. Repeated measures ANOVAs were performed using "time" (baseline and 6 and 12 months) as the within-subjects factor in the healthy control group. Wilcoxon signed-rank tests were used as post hoc tests to identify the time points at which the test scores were significantly different from those measured at baseline. We compared the scores of the surgical group with those of the nonsurgical group using repeated measures of the Mann-Whitney U-test at each time point. Finally, the effect of clinical symptom changes on neuropsychological performance was analyzed using Spearman's correlations to compare delta scores on the Y-BOCS and neuropsychological variables at the 12-month time point. All figures in this paper were created in GraphPad Prism 5.

TABLE 2. Neuropsychological domains, functions, and tests employed in this study

\begin{tabular}{|c|c|}
\hline Domain/Function & Test/Subtest \\
\hline Intellectual function & WASI ${ }^{44}$ \\
\hline Verbal intelligence & Similarities (for abstract verbal reasoning) \\
\hline Nonverbal intelligence & $\begin{array}{l}\text { Block Design (for visuospatial processing } \\
\text { capability) }\end{array}$ \\
\hline \multirow{2}{*}{$\begin{array}{l}\text { Verbal memory } \\
\text { functioning }\end{array}$} & WMS-R ${ }^{43}$ \\
\hline & $\begin{array}{l}\text { Immediate \& Delayed (30 minutes) Logical } \\
\text { Memory (for verbal memory capability) }\end{array}$ \\
\hline \multirow{2}{*}{$\begin{array}{l}\text { Nonverbal memory } \\
\text { functioning }\end{array}$} & WMS-R ${ }^{43}$ \\
\hline & $\begin{array}{l}\text { Immediate \& Delayed ( } 30 \text { minutes) Visual Re- } \\
\text { production (for visual memory capabilities) }\end{array}$ \\
\hline \multirow[t]{2}{*}{ Executive functioning } & WCST ${ }^{11}$ \\
\hline & $\begin{array}{l}\text { Corrects, Categories, Perseverative Errors, } \\
\text { Nonperseverative Errors, \& Errors (for set- } \\
\text { shifting \& decision-making capabilities) }\end{array}$ \\
\hline
\end{tabular}



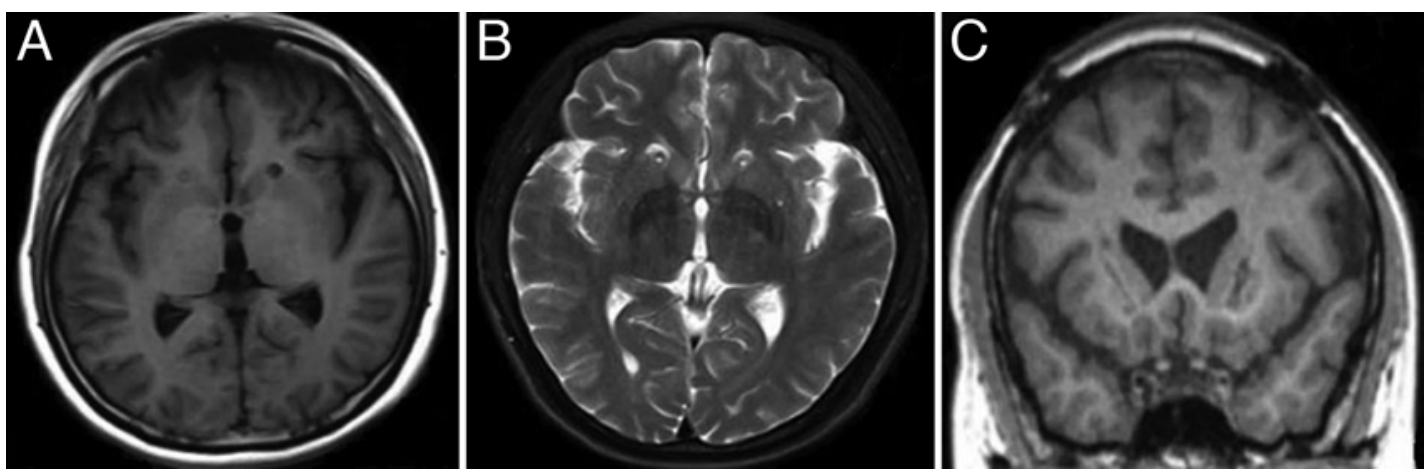

FIG. 1. Surgical Case 12. Typical results of bilateral $A C$ via thermocoagulation at 3 months after surgery. A: Axial T1-weighted MR image. B: Axial T2-weighted MR image. C: Coronal T1-weighted MR image.

\section{Results}

\section{Clinical Symptom Results}

Repeated measures ANOVA was performed on the YBOCS scores during the follow-up period (Tables 3 and 4). Patients who had undergone surgery exhibited clinical symptom improvements postoperatively. Significant decreases in the Y-BOCS scores were observed $\left(\chi^{2}=19.154\right.$, $\mathrm{p}=0.000$ ). Wilcoxon signed-rank tests were performed to determine the intervals at which these changes were statistically significant. As early as 1 month after surgery, the Y-BOCS scores showed significant improvement $(\mathrm{z}=$ $-3.297, p=0.001$ ) and these improvements appeared to be consistent. At the 12-month time point, the mean YBOCS score (10.21) was significantly less than the mean preoperative score $(29.21 ; \mathrm{z}=-3.235, \mathrm{p}=0.001)$. As shown in Fig. 2, surgical patients 8 and 9 (S8 and S9) exhibited Y-BOCS score reductions of less than $35 \%$ compared with their baseline scores. Surgical patient 8 resumed drug therapy 10 months after surgery, and S9 was lost to follow-up 15 months after surgery. Surgical patient 14 experienced symptom recurrence 8 months after surgery and underwent a second surgery 13 months after the first. But decreases in Y-BOCS scores were noted in the nonsurgical group as well $\left(\chi^{2}=5.794, p=0.000\right)$. Repeated measures Mann-Whitney U-tests were used to compare the Y-BOCS scores of surgical patients with those of the nonsurgical patients. Scores in the surgical group (after 1 month: $\mathrm{z}=$ $-2.809, \mathrm{p}=0.005$; after 12 months: $\mathrm{z}=-2.094, \mathrm{p}=0.036$ ) were significantly lower at each time point $(1,3,6$, and 12 months after surgery) except at baseline.

\section{Neuropsychological Testing Results in the Surgical Group}

Repeated measures ANOVA was also performed on all neuropsychological test scores during follow-up in the surgical group. As shown in Table 3, the surgical patients exhibited significant improvements on Block Design $\left(\chi^{2}=\right.$ $3.444, \mathrm{p}=0.013)$, Immediate Logical Memory $\left(\chi^{2}=4.204\right.$, $\mathrm{p}=0.004)$, Immediate Visual Reproduction $\left(\chi^{2}=6.558\right.$, $\mathrm{p}=0.000)$, Delayed Visual Reproduction $\left(\chi^{2}=4.611, \mathrm{p}=\right.$ $0.002)$, Correct $\left(\chi^{2}=5.009, \mathrm{p}=0.001\right)$, Errors $\left(\chi^{2}=5.009\right.$,

TABLE 3. Summary of neuropsychological changes in the surgical group throughout the 12-month follow-up period

\begin{tabular}{|c|c|c|c|c|c|c|}
\hline Test Name & Baseline & $1 \mathrm{Mo}$ & 3 Mos & 6 Mos & 12 Mos & $p$ Value \\
\hline Y-BOCS & $29.21(5.37)$ & $16.50(4.78)$ & $11.21(6.12)$ & $10.64(8.03)$ & $10.21(9.18)$ & 0.000 \\
\hline \multicolumn{7}{|l|}{ WASI } \\
\hline Similarities & $17.57(2.85)$ & $15.93(3.69)$ & $17.21(3.12)$ & $17.79(3.40)$ & $17.35(2.59)$ & 0.564 \\
\hline$B D$ & $31.50(10.76)$ & $31.71(9.57)$ & $36.07(6.72)$ & $39.43(7.20)$ & $40.36(6.87)$ & 0.013 \\
\hline \multicolumn{7}{|l|}{ WMS-R } \\
\hline ILM & $17.21(5.62)$ & $15.50(5.68)$ & $19.50(5.35)$ & $21.64(5.57)$ & $22.86(5.52)$ & 0.004 \\
\hline DLM & $14.79(6.10)$ & $14.36(5.97)$ & $17.57(6.85)$ & $18.43(6.24)$ & $20.00(6.00)$ & 0.092 \\
\hline IVR & $9.79(3.89)$ & $10.29(2.02)$ & $12.21(1.81)$ & $12.50(1.40)$ & $13.36(0.63)$ & 0.000 \\
\hline DVR & 8.57 (3.69) & $9.07(3.29)$ & $11.29(3.56)$ & 12.07 (2.09) & $12.21(1.67)$ & 0.002 \\
\hline \multicolumn{7}{|l|}{ WCST } \\
\hline Correct & $24.93(7.50)$ & $21.86(8.11)$ & $27.71(8.81)$ & $30.79(7.50)$ & $33.29(5.70)$ & 0.001 \\
\hline Errors & $23.07(7.50)$ & 26.14 (8.11) & $20.29(8.81)$ & $17.21(7.50)$ & $14.71(5.70)$ & 0.001 \\
\hline PEs & $16.86(9.53)$ & $20.21(10.30)$ & $13.43(12.23)$ & $10.14(8.44)$ & $6.86(5.86)$ & 0.004 \\
\hline NPEs & $6.21(2.36)$ & $5.93(2.97)$ & $7.29(3.97)$ & $7.07(2.20)$ & $7.86(1.96)$ & 0.351 \\
\hline Categories & $3.86(1.56)$ & $3.14(1.17)$ & $4.14(1.51)$ & $4.57(1.09)$ & $5.14(0.95)$ & 0.002 \\
\hline
\end{tabular}

$\mathrm{BD}=$ Block Design; DLM = Delayed Logical Memory; DVR = Delayed Visual Reproduction; ILM = Immediate Logical Memory; IVR = Immediate Visual Reproduction; NPE = Nonperseverative Errors; $P E=$ Perseverative Errors.

Values expressed as mean (standard deviation). 
TABLE 4. Summary of neuropsychological changes in the nonsurgical group throughout the 12-month follow-up period

\begin{tabular}{|c|c|c|c|c|c|c|}
\hline Test Name & Baseline & $1 \mathrm{Mo}$ & 3 Mos & 6 Mos & 12 Mos & p Value \\
\hline Y-BOCS & $30.78(6.48)$ & $23.42(7.42)$ & $20.92(8.25)$ & $19.50(9.08)$ & $16.64(9.96)$ & 0.000 \\
\hline \multicolumn{7}{|l|}{ WASI } \\
\hline Similarities & $17.42(2.73)$ & $17.00(3.16)$ & $16.85(2.59)$ & $17.00(2.77)$ & $17.92(2.33)$ & 0.835 \\
\hline$B D$ & $34.00(8.54)$ & $34.35(8.72)$ & $34.35(8.46)$ & $36.57(7.55)$ & $37.28(8.68)$ & 0.774 \\
\hline \multicolumn{7}{|l|}{ WMS-R } \\
\hline ILM & $15.35(7.01)$ & $15.90(6.92)$ & 17.57 (7.02) & $18.80(7.03)$ & $19.85(7.13)$ & 0.043 \\
\hline DLM & $11.78(6.32)$ & $12.14(5.97)$ & $10.92(4.89)$ & $13.57(6.93)$ & $13.28(6.21)$ & 0.775 \\
\hline IVR & $10.21(2.75)$ & $9.42(2.34)$ & $10.42(2.90)$ & $10.71(3.45)$ & $11.14(2.56)$ & 0.586 \\
\hline DVR & $10.35(2.73)$ & $9.92(1.63)$ & $10.21(2.54)$ & $10.42(2.47)$ & $11.07(2.40)$ & 0.782 \\
\hline \multicolumn{7}{|l|}{ WCST } \\
\hline Correct & $31.92(5.39)$ & $32.28(5.55)$ & $32.42(5.43)$ & $32.92(5.18)$ & $33.35(5.22)$ & 0.961 \\
\hline Error & $16.08(5.39)$ & $15.72(5.55)$ & $15.57(5.43)$ & $15.07(5.18)$ & $14.65(5.22)$ & 0.961 \\
\hline PE & $5.92(2.43)$ & $5.93(2.23)$ & $5.28(2.30)$ & $4.92(2.05)$ & $4.85(1.70)$ & 0.520 \\
\hline NPE & $10.16(2.25)$ & $9.79(2.56)$ & $10.29(2.57)$ & $10.15(2.58)$ & $9.80(1.77)$ & 0.612 \\
\hline Categories & $5.14(0.86)$ & $5.14(0.94)$ & $5.00(0.87)$ & $5.35(0.74)$ & $5.50(0.75)$ & 0.546 \\
\hline
\end{tabular}

$\mathrm{p}=0.001)$, Perseverative Errors $\left(\chi^{2}=4.326, \mathrm{p}=0.004\right)$, and Categories $\left(\chi^{2}=4.840, p=0.002\right)$ scores at the 12-month follow-up. Moreover, Wilcoxon signed-rank tests were performed to determine the intervals at which these improvements were statistically significant. Interestingly, compared with baseline, mild impairments in the Immediate Logical Memory $(\mathrm{z}=-2.017, \mathrm{p}=0.044)$ and Correct $(\mathrm{z}=-2.050, \mathrm{p}=0.040)$ scores were noted 1 month after surgery (Fig. 3). However, significant improvements in Block Design ( $\mathrm{z}=-2.201, \mathrm{p}=0.028)$, Immediate Logical Memory $(z=-2.677, p=0.007)$, Delayed Logical Memory $(\mathrm{z}=-2.619, \mathrm{p}=0.009)$, Immediate Visual Reproduction $(\mathrm{z}=-2.731, \mathrm{p}=0.006)$, and Delayed Visual Reproduction $(\mathrm{z}=-2.299, \mathrm{p}=0.021)$ performance were detected as early as 3 months after surgery. Regarding the Correct $(\mathrm{z}=-3.150, \mathrm{p}=0.002)$ and Errors $(\mathrm{z}=-3.150, \mathrm{p}=0.002)$ scores, improvements were first noted at the 6-month time point. Additionally, significant improvements in the Perse- verative Errors $(\mathrm{z}=-3.185, \mathrm{p}=0.001)$ and Categories $(\mathrm{z}=$ $-3.066, p=0.002)$ scores were first noted at the 12-month time point.

\section{Neuropsychological Testing Results in the Nonsurgical and Healthy Control Groups}

Repeated measures ANOVA was also performed on all neuropsychological test scores during the follow-up period in the nonsurgical and healthy control groups. As shown in Tables 4 and 5, significant improvements occurred only in the Immediate Logical Memory scores $\left(\chi^{2}=3.323\right.$, $\mathrm{p}=$ $0.043)$ in the nonsurgical group by the 12 -month followup. No significant differences were noted in any neuropsychological test scores in the healthy control group.

\section{Comparing Neuropsychological Test Scores Between the 2 OCD Groups and the Healthy Control Group}

Repeated measures Mann-Whitney U-tests were per-

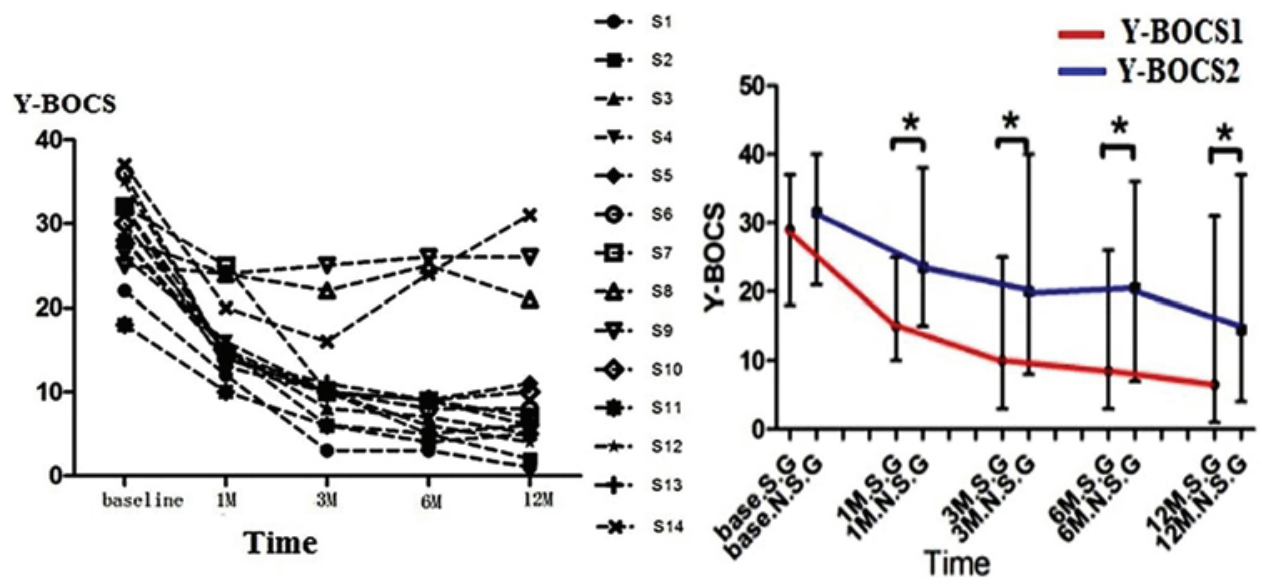

FIG. 2. Left: Y-BOCS score variations in 14 OCD patients who underwent surgery. Right: Differences in Y-BOCS score changes between the surgical and nonsurgical groups as a function of time. Base. = baseline; $M=$ month; N.S.G = nonsurgical group; $S 1, \ldots$ S14 = surgical patient $1, \ldots$ surgical patient 14; S.G = surgical group; Y-BOCS1 = Y-BOCS scores in surgical group; Y-BOCS2 = Y-BOCS scores in nonsurgical group. *Statistically significant differences. Figure is available in color online only. 


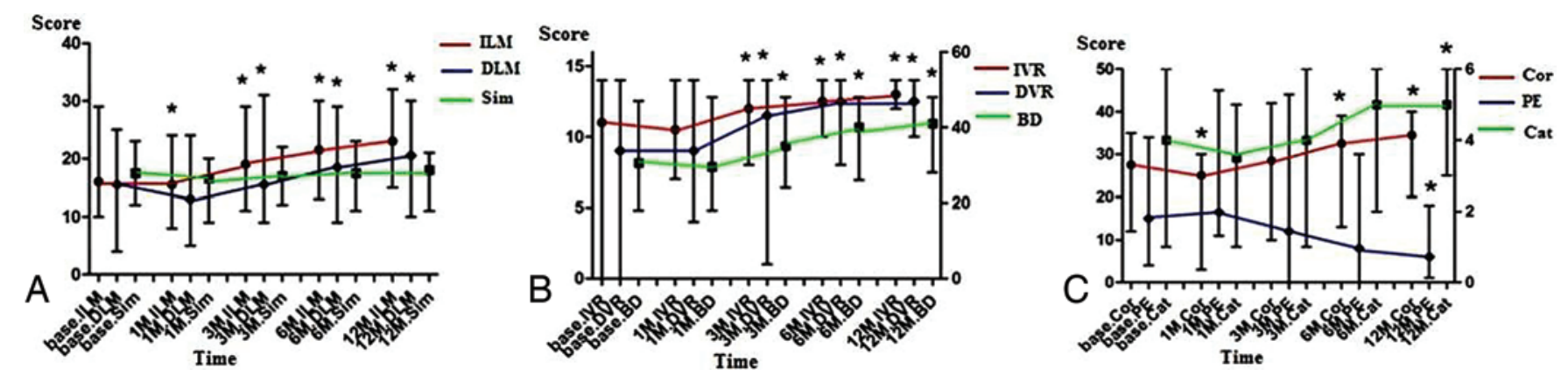

FIG. 3. Cognitive function changes in the surgical group over the 12-month follow-up. *Statistically significant differences. Base. = baseline; BD = Block Design; Cat = Categories; Cor = Correct; DLM = Delayed Logical Memory; DVR = Delayed Visual Reproduc tion; ILM = Immediate Logical Memory; IVR = Immediate Visual Reproduction; PEs = Perseverative Errors; Sim = Similarities. Figure is available in color online only.

formed to compare the scores of the surgical group with those of the nonsurgical and healthy control groups at baseline and at the 12-month time point. As shown in Fig. 4, at baseline, both the surgical and the nonsurgical groups scored significantly lower than the healthy control group on the Block Design (surgical vs control group: $\mathrm{z}=-2.119, \mathrm{p}=$ 0.034; nonsurgical vs control group: $\mathrm{z}=-1.981, \mathrm{p}=0.048$ ), Immediate Logical Memory $(\mathrm{z}=-2.354, \mathrm{p}=0.019 ; \mathrm{z}=$ $-2.576, p=0.010)$, Delayed Logical Memory $(z=-2.580$, $\mathrm{p}=0.010 ; \mathrm{z}=-3.168, \mathrm{p}=0.002)$, Immediate Visual Reproduction $(\mathrm{z}=-2.773, \mathrm{p}=0.006 ; \mathrm{z}=-3.040, \mathrm{p}=0.002)$, Delayed Visual Reproduction $(\mathrm{z}=-2.657, \mathrm{p}=0.008 ; \mathrm{z}$ $=-1.981, \mathrm{p}=0.048)$, Correct $(\mathrm{z}=-4.434, \mathrm{p}=0.000 ; \mathrm{z}=$ $-2.790, p=0.005)$, Perseverative Errors $(z=-4.272, p=$ $0.000 ; z=-3.475, p=0.001)$ tests. Compared with the scores in the healthy control group, the baseline Categories test scores were significantly lower in the surgical group $(\mathrm{z}$ $=-3.855, \mathrm{p}=0.000)$ but not in the nonsurgical group $(\mathrm{z}=$ $-1.742 \mathrm{p}=0.082)$. With the exception of the Correct ( $\mathrm{z}=$ $-2.720, p=0.007)$, Categories $(z=-2.743, p=0.006)$, and Perseverative Errors $(\mathrm{z}=-3.415, \mathrm{p}=0.001)$ scores, there were no significant differences in the neuropsychological tests scores between the surgical and nonsurgical groups.

As shown in Fig. 5, at the 12-month time point, differences in neuropsychological test scores between the surgical and healthy control groups were noted on the Correct $(\mathrm{z}=-2.791, \mathrm{p}=0.005)$, Categories $(\mathrm{z}=-2.818, \mathrm{p}=0.005)$, and Perseverative Errors $(\mathrm{z}=-2.808, \mathrm{p}=0.005)$ tests. Scores on the Immediate Logical Memory $(z=-2.165, p=$ $0.030)$, Delayed Logical Memory $(z=-2.716, p=0.007)$, Immediate Visual Reproduction $(\mathrm{z}=-3.370, \mathrm{p}=0.001)$ and Block Design $(\mathrm{z}=-1.985, \mathrm{p}=0.047)$ tests were significantly higher in the surgical group than in the nonsurgical group. The nonsurgical group scored significantly lower than the healthy controls on the Immediate Logical Memory $(z=-2.812, p=0.005)$, Delayed Logical Memory $(\mathrm{z}=-3.438, \mathrm{p}=0.001)$, Immediate Visual Reproduction $(\mathrm{z}$ $=-2.923, \mathrm{p}=0.003)$, Delayed Visual Reproduction $(\mathrm{z}=$ -2.157, $\mathrm{p}=0.031)$, Block Design $(\mathrm{z}=-2.031, \mathrm{p}=0.042)$, Correct $(z=-2.890, p=0.004)$, and Perseverative Errors $(\mathrm{z}=-3.660, \mathrm{p}=0.000)$ tests.

\section{Correlation Between Neuropsychological Changes and Symptom Improvements in the Surgical Group}

Spearman's correlation tests were performed to com- pare delta scores pertaining to clinical symptom changes with neuropsychological variables in the surgical group. As shown in Fig. 6, of all the delta scores recorded between baseline and 12 months after surgery in the surgical group, only the delta score for the Delayed Logical Memory test significantly correlated with the delta score for the Y-BOCS test $(r=0.65, p=0.012)$. The other delta scores, including those for the Block Design $(r=0.343, p=$ $0.230)$, Immediate Logical Memory $(r=0.183, p=0.532)$, Immediate Visual Reproduction $(\mathrm{r}=-0.194, \mathrm{p}=0.506)$, Delayed Visual Reproduction ( $r=-0.061, p=0.837)$, Correct $(\mathrm{r}=-0.251, \mathrm{p}=0.387)$, Categories $(\mathrm{r}=-0.08, \mathrm{p}=$ 0.786), and Perseverative Errors ( $\mathrm{r}=0.41, \mathrm{p}=0.146)$ tests, did not significantly correlate with the delta score for the Y-BOCS test.

\section{Side Effects in Surgical Patients}

Most of the adverse events experienced by patients were transient. Two patients $(\mathrm{S} 10, \mathrm{~S} 14)$ experienced incontinence for the first 2-3 days after surgery. Six patients (S2, S3, S6, S9, S10, S11) experienced hypersomnia for a few days after surgery. Six patients (S1, S4, S7, S8, S9, S14) exhib-

TABLE 5. Summary of neuropsychological changes in the healthy control group throughout the 12-month follow-up period

\begin{tabular}{ccccc}
\hline Test Name & Baseline & 6 Mos & 12 Mos & p Value \\
\hline WASI & & & & \\
\hline Similarities & $17.78(1.52)$ & $17.92(2.86)$ & $18.57(1.94)$ & 0.603 \\
\hline BD & $39.78(7.07)$ & $39.43(7.20)$ & $40.36(6.87)$ & 0.939 \\
\hline WMS-R & & & & \\
\hline ILM & $24.28(8.19)$ & $24.35(7.15)$ & $25.92(6.63)$ & 0.801 \\
\hline DLM & $22.21(8.04)$ & $22.42(6.89)$ & $23.78(6.85)$ & 0.826 \\
\hline IVR & $12.85(1.70)$ & $13.07(1.07)$ & $13.21(1.18)$ & 0.781 \\
\hline DVR & $12.00(2.80)$ & $12.00(2.44)$ & $12.57(1.94)$ & 0.773 \\
\hline WCST & & & & \\
\hline Correct & $36.00(1.96)$ & $37.07(2.81)$ & $38.50(2.95)$ & 0.051 \\
\hline Error & $12.00(1.96)$ & $10.93(2.81)$ & $9.50(2.95)$ & 0.051 \\
\hline PE & $2.07(2.70)$ & $2.07(2.09)$ & $1.78(1.92)$ & 0.929 \\
\hline NPE & $9.93(2.45)$ & $8.86(2.44)$ & $7.72(2.52)$ & 0.601 \\
\hline Categories & $5.85(0.36)$ & $5.78(0.42)$ & $5.92(0.26)$ & 0.577 \\
\hline
\end{tabular}



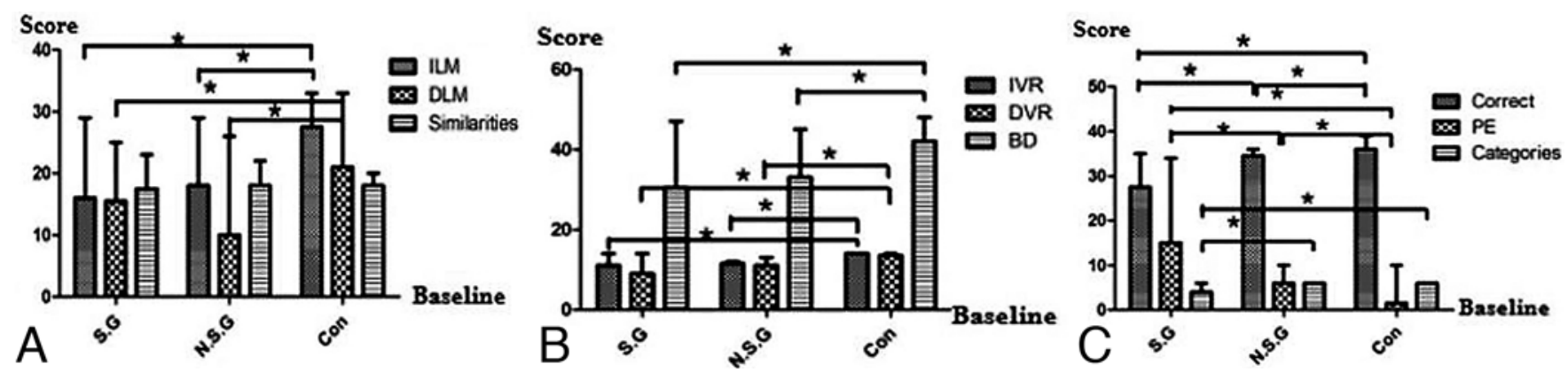

FIG. 4. Differences in cognitive function among the 3 groups at baseline. *Statistically significant differences. BD = Block Design; Con = healthy control group; DLM = Delayed Logical Memory; DVR = Delayed Visual Reproduction; ILM = Immediate Logical Memory; IVR = Immediate Visual Reproduction; N.S.G = nonsurgical group; PE = Perseverative Errors; S.G = surgical group.

ited various degrees of childish behavior, but most of them experienced symptom resolution within 3 months, and 1 patient (S7) experienced complete symptom resolution at 10 months after surgery. Four patients (S4, S7, S10, S12) experienced occasional visual hallucinations during the 1st month after surgery. All patients experienced symptom remission upon receiving appropriate pharmacological therapy. Two patients exhibited symptoms of sexual disinhibition; one (S9) experienced symptom resolution 6 months after surgery, while the other (S14) began exhibiting symptoms of severe sexual disinhibition 8 months after surgery, as well as OCD symptom recurrence. Ultimately, this patient underwent a second surgery 13 months after her first. Three patients (S7, S13, S14) exhibited personality changes, including lack of interest, inappropriate laughter, and decreased motivation, which proved to be permanent at the 12-month time point. Weight gain, which has often been reported in similar studies, was not observed in this study.

\section{Discussion}

Findings in the present study suggest that improvements in clinical symptoms can appear as early as 1 month after surgery and can be sustained thereafter. Eleven (78.6\%) of 14 patients responded to therapy within 12 months. Our results are consistent with those of previous studies. ${ }^{16,21,28} \mathrm{In}$ our limited experience, $\mathrm{AC}$ is effective at reducing $\mathrm{OCD}$ symptoms. However, this procedure is associated with several (reversible) adverse effects, such as personality chang- es, childish behavior, visual hallucinations, incontinence, hypersomnia, and sexual disinhibition.

Interestingly, the nonsurgical OCD group exhibited appropriate improvements in the Y-BOCS scores. This group received systematic drug therapy and CBT during the 12-month follow-up period, which may explain these improvements. According to several previous studies, both SSRIs and CBT are effective at reducing symptoms in patients with treatment-refractory OCD., ${ }^{2,31}$ Furthermore, although the 2 OCD groups matched in terms of their initial Y-BOCS scores, they did not match in terms of baseline obsessive-compulsive symptom duration or SSRI treatment duration, as both durations were shorter in the surgical OCD group, which may explain the above results. However, the placebo effect of multiple visits may have also affected our results, an unavoidable and important shortcoming of this study.

Our goal in the present study was to investigate changes in OCD patients' cognitive function over a 12-month postoperative follow-up period. Thus, our study was divided into 3 stages. First, we defined the cognitive deficits that characterize treatment-refractory OCD. Next, we investigated how cognitive function changes with time in OCD patients after surgery and compared these changes with those experienced by nonsurgical OCD patients and healthy controls. Lastly, we explored how AC modifies cognitive performance in treatment-refractory OCD patients and determined whether posttreatment cognitive improvements are related to OCD symptom alleviation.
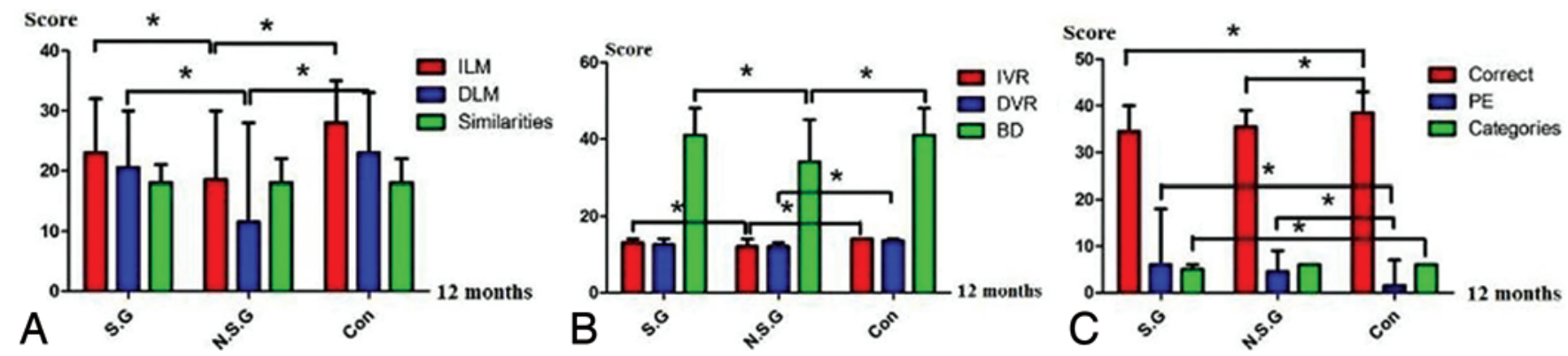

FIG. 5. Differences in cognitive function among the 3 groups at 12 months after surgery. *Statistically significant differences. BD = Block Design; Con = healthy control group; DLM = Delayed Logical Memory; DVR = Delayed Visual Reproduction; ILM = Immediate Logical Memory; IVR = Immediate Visual Reproduction; N.S.G = nonsurgical group; PE = Perseverative Errors; S.G = surgical group. Figure is available in color online only. 


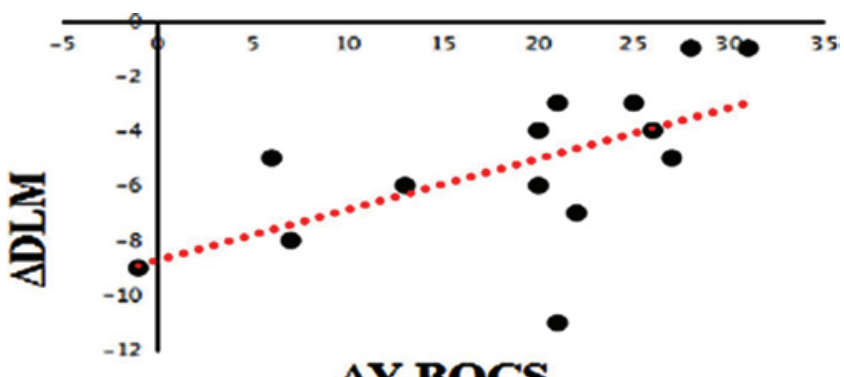

$\triangle \mathrm{Y}$-BOCS

FIG. 6. Correlation between Y-BOCS delta scores and Delayed Logical Memory before surgery and 12 months after surgery. $\triangle \mathrm{DLM}=$ delta scores of the Delayed Logical Memory subtest; $\triangle \mathrm{Y}$-BOCS $=$ delta scores of the Y-BOCS. Figure is available in color online only.

\section{Cognitive Deficits in OCD Patients Before Surgery}

A large number of studies on the neuropsychological deficits that characterize OCD have been conducted. In the past 25 years, researchers have explored several cognitive domains, including memory (for example, verbal memory $\mathrm{y}^{4,37}$ and visual and spatial memory ${ }^{19,29,32,33}$ ) and executive function (for example, set shifting, , $22,23,27$ fluency, ${ }^{13,19,39}$ and decision making $\left.{ }^{5,14,35}\right)$. The majority of these studies have noted some degree of impairment in nonverbal memory, visuospatial skills, and visual and selective attention among OCD patients. , $^{6,24,36,38,41}$

We found that both surgical and nonsurgical OCD groups exhibited significantly impaired visuospatial processing, visual memory, verbal memory, and executive function at baseline as compared to healthy controls. These findings are consistent with those of most previous reports. Interestingly, before surgery the surgical group exhibited more severe executive function impairment than the nonsurgical group. As shown in Table 1, these 2 groups exhibited no significant differences in sex, age, education level, CBT duration, or Y-BOCS scores; however, there were significant differences in disease duration and SSRI treatment duration. This suggests that the degree of impairment in several advanced cognitive functions (such as executive function) is related to OCD symptom duration or drug treatment duration rather than symptom severity.

\section{Changes in Cognitive Function as a Result of $A C$ for Treatment-Refractory OCD}

We noted mild impairments in performance on most neuropsychological tests, particularly the Immediate Logical Memory and Correct subtests, 1 month after surgery compared with baseline, which we attributed to the destructive nature of AC. However, this phenomenon proved to be transient. Thereafter, visuospatial processing, visual memory, and verbal memory performance improved significantly compared with baseline. Moreover, these improvements persisted such that there were no significant differences between surgical patients and healthy controls at the 12-month follow-up. However, although the level of executive function exhibited by the surgical group eventually reached that exhibited by the nonsurgical group, it remained poorer than that exhibited by the healthy control group at 12 months after surgery. Importantly, neuropsy- chological improvements were observed across the following domains: visuospatial processing, visual memory, verbal memory, and executive function. These findings are in accordance with those of several recent studies. ${ }^{3,7,42}$ However, we did not observe any significant differences in abstract verbal reasoning ability (Similarities subtest) among the 3 groups before or after surgery. This finding is inconsistent with the results of Taub et al., ${ }^{42}$ who reported increases in the Similarities subtest scores in 4 of 5 refractory OCD patients after VC/VS gamma capsulotomy.

\section{Verbal Memory, Visual Memory, and Visuospatial Skill Changes in OCD Patients After AC}

In our study, verbal memory, visual memory, and visuospatial skills improved at the 3-month follow-up compared with baseline and reached normal levels by the last followup. Regarding verbal memory, our results are consistent with those of the following 2 studies. Taub et al ${ }^{42}$ reported improvements in Immediate and Delayed Logical Memory in 4 of 5 and 5 of 5 refractory OCD patients, respectively, following VC/VS gamma capsulotomy. However, that study included a small sample of participants who were evaluated at different time points. Batistuzzo et al. ${ }^{3}$ also noted a significant improvement in Immediate and Delayed Logical Memory test results in all OCD patients at 1 year after VC/VS gamma capsulotomy. However, that study did not include a healthy control group.

Regarding visual memory and visuospatial skill, Nyman et al. ${ }^{25}$ found that patients performed no better than the control patients on the Rey Complex Figure Test (testing visuospatial memory abilities) after capsulotomy. Taub et al. ${ }^{42}$ noted score increases on the Block Design subtest in only 2 of 5 refractory OCD patients after VC/VS gamma capsulotomy. Kim et al. ${ }^{13}$ showed that immediate recall on the Rey Complex Figure Test improved more in OCD patients than in control subjects over a period of 4 months. Batistuzzo et al. ${ }^{3}$ found that visuospatial memory performance improved significantly in an actively treated group $(\mathrm{n}=8)$ after GVC radiosurgery. Our visuospatial memory subtest results are consistent with those of Batistuzzo et al. ${ }^{3}$ and Kim et al. ${ }^{13}$ but are inconsistent with those of $\mathrm{Ny}-$ man et al. ${ }^{25}$ and Taub et al. ${ }^{42}$

It is well known that the prefrontal cortex is closely associated with memory. In particular, visuospatial memory is mediated by the orbitofrontal cortex and closely connected to the limbic area. ${ }^{8,15,30}$ Additionally, visuospatial memory deficits are often reported in OCD patients. A large body of research supports the idea that orbitofrontalstriatal circuits are a neuropathophysiological mechanism underlying OCD development. A recent meta-analysis of neuropsychological data pertaining to OCD patients indicated that visuospatial memory exhibits the most consistent cognitive improvements in OCD patients after treatment. ${ }^{40}$ It is possible that postoperative improvements in verbal memory, visual memory, and visuospatial skills occur secondary to improvements in obsessive-compulsive symptoms. Upon symptom improvement, patient concentration levels can spontaneously improve, resulting in gradual improvements in verbal memory and visuospatial memory. However, we noted similar improvements in 2 $(\mathrm{S} 8, \mathrm{~S} 9)$ patients without therapy response. 


\section{Executive Function Changes in OCD Patients After AC}

Several studies have reported that patients exhibit impaired performance in the Perseverative Errors and Responses categories on the WCST after Nyman and Mindus $^{26}$ noted that 3 of 5 patients presented with more perseverative responses 1 year postcapsulotomy. Rück et al. ${ }^{34}$ found that limited numbers of patients who had undergone preoperative and postoperative neuropsychological evaluations had poorer postoperative WCST results and concluded that capsulotomy induced mild executive function impairment in these patients by the long-term follow-up.

However, several studies have reported contrasting findings. Nyman et al..$^{25}$ noted that in a subgroup of capsulotomy patients who did not complete all 6 categories of the WCST, the number of years elapsed after capsulotomy correlated positively with the number of categories completed. Csigó et al. ${ }^{7}$ noted improvements in the majority of the abovementioned cognitive functions after AC, including improvements in several executive functions, such as set shifting (Stroop Test, California Sorting Test) and decision making (Iowa Gambling Test), during a 24-month follow-up. Batistuzzo et al. ${ }^{3}$ found that an actively treated group demonstrated significant improvements in WCST performance compared with a sham-treated group during a 12-month RCT.

Our results partially support those of previous studies. ${ }^{3,7,25}$ Our data indicate that executive function improved by the 6 -month and 12-month follow-ups, albeit at a slower rate than the OCD symptoms and memory. However, executive function did not reach a normal level at the 12-month time point. We surmised that advanced cognitive functions, such as executive functions, may require longer recovery periods. This hypothesis is supported by the findings of Nyman et al., ${ }^{25}$ who noted that the number of years elapsed after capsulotomy correlates positively with the number of categories completed on the WCST. Therefore, we will subject surgically treated patients to a much longer follow-up period in future studies. We believe that executive function performance can reach near-normal levels within a few years after AC.

\section{Variations in Cognitive Function Between Surgical and Nonsurgical Patients at the 12-Month Follow-Up}

The surgical group exhibited greater improvements in verbal memory, visual memory, visuospatial skills, and executive function than the nonsurgical group at the 12-month time point. These findings indicate that AC may be more effective than CBT or SSR Is at improving cognitive deficits in treatment-refractory OCD patients. However, the 2 groups of OCD patients in our study differed significantly regarding disease duration and SSRI treatment duration. Therefore, caution is warranted regarding the interpretation of our results.

\section{How Does AC Facilitate Cognitive Profile Changes in Treatment-Refractory OCD Patients?}

Milad and Rauch ${ }^{20}$ described the different components of the so-called cortico-striato-thalamo-cortical pathways commonly implicated in the psychopathology of OCD.
These pathways include the following 3 loops: an affective circuit, reflecting affective and reward processing: "anterior cingulate cortex/ventromedial prefrontal cortexnucleus accumbens-thalamus-cortex"; a dorsal cognitive circuit, reflecting working memory and executive function: "dorsolateral prefrontal cortex-dorsal caudate-thalamus-cortex"; and a ventral cognitive circuit, reflecting motor and response inhibition: "anterolateral orbitofrontal cortex-putamen-thalamus-cortex."

These loops interact with and are linked to one another. Studies have implicated the affective and ventral cognitive circuits in OCD. As a result, capsulotomy, cingulotomy, and nucleus accumbens deep brain stimulation procedures have been widely applied in the management of treatmentrefractory OCD and have often successfully attenuated obsessive-compulsive symptoms. Given the interconnectedness of these 3 loops, we hypothesize that OCD-related cognitive deficits are caused by the influence of the affective and ventral cognitive circuits and that targeting the internal capsule may affect the connections between the orbitofrontal cortex or ventromedial prefrontal cortex and the thalamus, interrupting the hyperfunctional frontal-striatal circuits associated with the pathophysiology of OCD, restoring dorsal cognitive circuit function ${ }^{18}$ and facilitating improvements not only in obsessive-compulsive symptoms but also in memory and executive function after AC. Additionally, because the dorsal caudate is located medial and ventral to the anterior limb of the internal capsule, it is possible that the memory and executive function impairments reported for previous studies ${ }^{26,34}$ are caused by large lesions resulting in damage to the dorsal cognitive circuit. Thus, accurate target location is vital to the success of AC. In our limited experience, we have found that smaller lesions are associated with lower risks of adverse effects and that lateral or medial lesions involving the anterior limb of the internal capsule are associated with better results. These findings are consistent with those of Rück et al., ${ }^{34}$ who found that OCD symptom reduction may be facilitated by reducing lateral lesion extension and that adverse effects may be minimized by reducing medial and posterior lesion extension. Taub et al. ${ }^{42}$ found that smaller, more ventrally located internal capsule lesions may have a different impact on cognitive outcomes. Lastly, we hypothesize that the projections of the dorsal cognitive circuit may pass close to the posterior region of the anterior limb of the internal capsule. This idea warrants further research, possibly diffusion tensor imaging-based research. Furthermore, we believe that improvements in cognitive performance are facilitated by improvements in obsessive-compulsive symptoms rather than interruptions of abnormal circuitry, as postulated by Batistuzzo et al. ${ }^{3}$ The Delayed Logical Memory subtest results exhibited a significant correlation with the Y-BOCS results in this study and may support the abovementioned idea. However, we did not note this outcome on other tests, perhaps because the sample was relatively small. Further research is warranted to elucidate the exact mechanisms underlying neuropsychological improvements post-OCD surgery.

\section{Study Limitations}

The findings of this study should be interpreted with 
caution for several reasons. First, the sample was relatively small (only 14 subjects in each group). Second, the 12-month follow-up period may not have been sufficient to observe improvements in specific cognitive functions, such as executive function. Third, given our strict inclusion and exclusion criteria, our results may not be generalizable to other populations. Fourth, we used relatively few neuropsychological tests, which may not have reflected all the cognitive deficits in our sample. Last, our results were clearly influenced by a placebo effect, particularly our Y-BOCS score results, most likely because of our use of short follow-up time intervals, such as the interval between the 1- and 3-month time points. To attenuate the short-term placebo effect exerted by repeated tests, we considered evaluating patients every 3 months after surgery. However, given our experience, we determined that the 1-month time point is important with respect to changes in OCD patient symptoms and cognitive function and thus included this time point in our study. We also tried to use different versions of specific OCD symptom scales at different time points to minimize the placebo effect; however, no appropriate scales are available to replace the Y-BOCS. Therefore, we used this scale at each time point, which means that the 2 OCD groups experienced the same placebo effect. We used symptom and cognitive function analyses to determine if the placebo effect significantly influenced our results. We surmised that if the placebo effect had been significant, the results noted in the 2 OCD groups at each time point would be similar because the initial symptoms and cognitive function deficits noted in these groups were similar. However, we found that the 2 OCD groups exhibited significant differences with respect to symptoms and cognitive function, indicating that the placebo effect did not significantly influence our results. Nonetheless, we were unable to determine the exact degree to which the placebo effect did influence our results. This was an important shortcoming of our study, although we do not believe that the placebo effect significantly affected its outcome. In future studies, we will attempt to use follow-up intervals of 3 months, 6 months, or longer, as well as different versions of the same performance tests, to minimize the placebo effect. Furthermore, there were significant differences between the surgical and nonsurgical OCD groups (disease duration and SSRI treatment duration) at baseline, which may have influenced the test performance of each group. Despite these limitations, we believe that our findings are both clinically and scientifically valuable because of the very small number of patients who received the treatment described herein. In the future, we will expand our sample size, use more neuropsychological tests, and evaluate patients over a much longer follow-up period.

\section{Conclusions}

Our data suggest that $\mathrm{AC}$ does not give rise to cognitive deficits as we observed significant improvements in verbal memory, visuospatial memory, and executive function, which may have been secondary to OCD symptom amelioration.

\section{References}

1. Abbruzzese M, Ferri S, Scarone S: Wisconsin Card Sorting Test performance in obsessive-compulsive disorder: no evidence for involvement of dorsolateral prefrontal cortex. Psychiatry Res 58:37-43, 1995

2. Abramowitz JS, Franklin ME, Schwartz SA, Furr JM: Symptom presentation and outcome of cognitive-behavioral therapy for obsessive-compulsive disorder. J Consult Clin Psychol 71:1049-1057, 2003

3. Batistuzzo MC, Hoexter MQ, Taub A, Gentil AF, Cesar RC, Joaquim MA, et al: Visuospatial memory improvement after gamma ventral capsulotomy in treatment refractory obsessive-compulsive disorder patients. Neuropsychopharmacology 40:1837-1845, 2015

4. Boone KB, Ananth J, Philpott L, Kaur A, Djenderedjian A: Neuropsychological characteristics of nondepressed adults with obsessive-compulsive disorder. Cogn Behav Neurol 4:96-109, 1991

5. Cavallaro R, Cavedini P, Mistretta P, Bassi T, Angelone SM, Ubbiali A, et al: Basal-corticofrontal circuits in schizophrenia and obsessive-compulsive disorder: a controlled, double dissociation study. Biol Psychiatry 54:437-443, 2003

6. Cohen LJ, Hollander E, DeCaria CM, Stein DJ, Simeon D, Liebowitz MR, et al: Specificity of neuropsychological impairment in obsessive-compulsive disorder: a comparison with social phobic and normal control subjects. J Neuropsych Clin N 8:82-85, 1996

7. Csigó K, Harsányi A, Demeter G, Rajkai C, Németh A, Racsmány M: Long-term follow-up of patients with obsessivecompulsive disorder treated by anterior capsulotomy: a neuropsychological study. J Affect Disord 126:198-205, 2010

8. Frey S, Petrides M: Orbitofrontal cortex and memory formation. Neuron 36:171-176, 2002

9. Goodman WK, Price LH, Rasmussen SA, Mazure C, Fleischmann RL, Hill CL, et al: The Yale-Brown Obsessive Compulsive Scale. I. Development, use, and reliability. Arch Gen Psychiatry 46:1006-1011, 1989

10. Head D, Bolton D, Hymas N: Deficit in cognitive shifting ability in patients with obsessive-compulsive disorder. Biol Psychiatry 25:929-937, 1989

11. Heaton RK, Chelune G, Talley J, Kay G, Curtiss G: Wisconsin Card Sorting Test Manual-Revised and Expanded. Odessa: Psychological Assessment Resourses, 1993

12. Husted DS, Shapira NA: A review of the treatment for refractory obsessive-compulsive disorder: from medicine to deep brain stimulation. CNS Spectr 9:833-847, 2004

13. Kim MS, Park SJ, Shin MS, Kwon JS: Neuropsychological profile in patients with obsessive-compulsive disorder over a period of 4-month treatment. J Psychiatr Res 36:257-265, 2002

14. Lawrence NS, Wooderson S, Mataix-Cols D, David R, Speckens A, Phillips ML: Decision making and set shifting impairments are associated with distinct symptom dimensions in obsessive-compulsive disorder. Neuropsychology 20:409-419, 2006

15. Lepage M, McIntosh AR, Tulving E: Transperceptual encoding and retrieval processes in memory: a PET study of visual and haptic objects. Neuroimage 14:572-584, 2001

16. Liu K, Zhang H, Liu C, Guan Y, Lang L, Cheng Y, et al: Stereotactic treatment of refractory obsessive compulsive disorder by bilateral capsulotomy with 3 years follow-up. J Clin Neurosci 15:622-629, 2008

17. Lopes AC, de Mathis ME, Canteras MM, Salvajoli JV, Del Porto JA, Miguel EC: [Update on neurosurgical treatment for obsessive compulsive disorder.] Rev Bras Psiquiatr 26:6266, 2004 (Portuguese)

18. Lopes AC, Greenberg BD, Norén G, Canteras MM, Busatto GF, de Mathis ME, et al: Treatment of resistant obsessivecompulsive disorder with ventral capsular/ventral striatal 
gamma capsulotomy: a pilot prospective study. J Neuropsychiatry Clin Neurosci 21:381-392, 2009

19. Martinot JL, Allilaire JF, Mazoyer BM, Hantouche E, Huret JD, Legaut-Demare F, et al: Obsessive-compulsive disorder: a clinical, neuropsychological and positron emission tomography study. Acta Psychiatr Scand 82:233-242, 1990

20. Milad MR, Rauch SL: Obsessive-compulsive disorder: beyond segregated cortico-striatal pathways. Trends Cogn Sci 16:43-51, 2012

21. Mindus P, Meyerson BA: Anterior capsulotomy for intractable anxiety disorders, in Schmidek HH, Sweet WH (eds): Schmidek \& Sweet Operative Neurosurgical Techniques: Indications, Methods, and Results, ed 3. Philadelphia: Saunders, 1995, pp 1413-1421

22. Moritz S, Birkner C, Kloss M, Jacobsen D, Fricke S, Böthern A, et al: Impact of comorbid depressive symptoms on neuropsychological performance in obsessive-compulsive disorder. J Abnorm Psychol 110:653-657, 2001

23. Moritz S, Birkner C, Kloss M, Jahn H, Hand I, Haasen C, et al: Executive functioning in obsessive-compulsive disorder, unipolar depression, and schizophrenia. Arch Clin Neuropsychol 17:477-483, 2002

24. Nelson E, Early TS, Haller JW: Visual attention in obsessivecompulsive disorder. Psychiatry Res 49:183-196, 1993

25. Nyman H, Andréewitch S, Lundbäck E, Mindus P: Executive and cognitive functions in patients with extreme obsessivecompulsive disorder treated by capsulotomy. Appl Neuropsychol 8:91-98, 2001

26. Nyman H, Mindus P: Neuropsychological correlates of intractable anxiety disorder before and after capsulotomy. Acta Psychiatr Scand 91:23-31, 1995

27. Okasha A, Rafaat M, Mahallawy N, El Nahas G, El Dawla AS, Sayed M, et al: Cognitive dysfunction in obsessive-compulsive disorder. Acta Psychiatr Scand 101:281-285, 2000

28. Oliver B, Gascón J, Aparicio A, Ayats E, Rodriguez R, Maestro De León JL, et al: Bilateral anterior capsulotomy for refractory obsessive-compulsive disorders. Stereotact Funct Neurosurg 81:90-95, 2003

29. Penadés R, Catalán R, Andrés S, Salamero M, Gastó C: Executive function and nonverbal memory in obsessive-compulsive disorder. Psychiatry Res 133:81-90, 2005

30. Petrides M, Alivisatos B, Frey S: Differential activation of the human orbital, mid-ventrolateral, and mid-dorsolateral prefrontal cortex during the processing of visual stimuli. Proc Natl Acad Sci U S A 99:5649-5654, 2002

31. Pigott TA, Seay SM: A review of the efficacy of selective serotonin reuptake inhibitors in obsessive-compulsive disorder. J Clin Psychiatry 60:101-106, 1999

32. Purcell R, Maruff P, Kyrios M, Pantelis C: Cognitive deficits in obsessive-compulsive disorder on tests of frontal-striatal function. Biol Psychiatry 43:348-357, 1998

33. Purcell R, Maruff P, Kyrios M, Pantelis C: Neuropsychological deficits in obsessive-compulsive disorder: a comparison with unipolar depression, panic disorder, and normal controls. Arch Gen Psychiatry 55:415-423, 1998

34. Rück C, Karlsson A, Steele JD, Edman G, Meyerson BA, Ericson K, et al: Capsulotomy for obsessive-compulsive disorder: long-term follow-up of 25 patients. Arch Gen Psychiatry 65:914-921, 2008

35. Sachdev P, Hay P: Does neurosurgery for obsessive-compul- sive disorder produce personality change? J Nerv Ment Dis 183:408-413, 1995

36. Savage CR, Baer L, Keuthen NJ, Brown HD, Rauch SL, Jenike MA: Organizational strategies mediate nonverbal memory impairment in obsessive-compulsive disorder. Biol Psychiatry 45:905-916, 1999

37. Savage CR, Deckersbach T, Wilhelm S, Rauch SL, Baer L, Reid T, et al: Strategic processing and episodic memory impairment in obsessive compulsive disorder. Neuropsychology 14:141-151, 2000

38. Savage CR, Keuthen NJ, Jenike MA, Brown HD, Baer L, Kendrick AD, et al: Recall and recognition memory in obsessive-compulsive disorder. J Neuropsychiatry Clin Neurosci 8:99-103, 1996

39. Schmidtke K, Schorb A, Winkelmann G, Hohagen F: Cognitive frontal lobe dysfunction in obsessive-compulsive disorder. Biol Psychiatry 43:666-673, 1998

40. Shin NY, Lee TY, Kim E, Kwon JS: Cognitive functioning in obsessive-compulsive disorder: a meta-analysis. Psychol Med 44:1121-1130, 2014

41. Tallis F, Pratt P, Jamani N: Obsessive compulsive disorder, checking, and non-verbal memory: a neuropsychological investigation. Behav Res Ther 37:161-166, 1999

42. Taub A, Lopes AC, Fuentes D, D’Alcante CC, de Mathis ME, Canteras MM, et al: Neuropsychological outcome of ventral capsular/ventral striatal gamma capsulotomy for refractory obsessive-compulsive disorder: a pilot study. J Neuropsychiatry Clin Neurosci 21:393-397, 2009

43. Wechsler D: Manual for the Wechsler Memory Scale-Revised. San Antonio: The Psychological Corporation, 1987

44. Wechsler D: Wechsler Abbreviated Scale of Intelligence. San Antonio: Psychological Corporation, 1999

\section{Disclosures}

The authors report no conflict of interest concerning the materials or methods used in this study or the findings specified in this paper.

\section{Author Contributions}

Conception and design: Wang, P Li. Acquisition of data: Gong, B Li, S Zhang, X Zhang, Yang, Liu. Analysis and interpretation of data: Gong. Drafting the article: Gong, P Li. Critically revising the article: all authors. Reviewed submitted version of manuscript: all authors. Statistical analysis: S Zhang. Study supervision: Wang, P Li.

\section{Supplemental Information \\ Previous Presentations}

Portions of this work were presented in abstract form at the American Society for Stereotactic and Functional Neurosurgery 2016 meeting held in Chicago, Illinois, on June 18-21, 2016.

\section{Correspondence}

Wei Wang, Department of Neurosurgery, West China Hospital, Sichuan University, 37 Guoxue Alley, Chengdu, Sichuan Province 610041, People's Republic of China. email: wcnsww@163.com. 